Pacific Journal of 


\title{
ON THE SPECTRAL ANALYSIS OF BOUNDED FUNCTIONS
}

\author{
PAUL Koosis
}

The spectral analysis of functions $f \in L_{\infty}(-\infty, \infty)$, and the different possible ways of looking at it, are discussed. A method is given for constructing functions $f \in L_{\infty}(-\infty, \infty)$ having the property that 0 is in the spectrum of $f$, but 1 is not the weak limit of any sequence of linear combinations of translates of $f$.

Preliminary discussion. Let $f \in L_{\infty}(-\infty, \infty)$. As is customary, we say that a real number $\lambda$ belongs to the spectrum of $f$, and write $\lambda \in s p f$, when the function $e^{i \lambda x}$ is in the weakly closed (i.e., closed in the weak topology over $L_{1}$ ) subspace of $L_{\infty}$ generated by the translates of $f$. (A translate of $f$ is any function $f_{h}$ of the form $f_{h}(x)=f(x-h)$ for $-\infty<h<\infty$.) As an immediate consequence of Wiener's Tauberian theorem, one has, by duality (see, for instance [5], p. 128, [9], p. 185 , [4], p. 106 or [7], p. 181) the

Theorem on Spectral Analysis. Unless $f \in L_{\infty}$ is zero almost everywhere, sp $f$ is nonempty.

This result is often referred to as Beurling's theorem. However, Beurling proved much more about a smaller class of functions $f$. If the $\psi_{n}(n=1,2, \cdots)$ and $\psi$ are bounded continuous functions on $(-\infty, \infty)$, let us agree to say that $\psi_{n} \rightarrow \psi$ narrowly as $n \rightarrow \infty$ if $\left\|\psi_{n}\right\|_{\infty} \rightarrow\|\psi\|_{\infty}$ and $\psi_{n}(x) \rightarrow \psi(x)$ uniformly on finite intervals for $n \rightarrow \infty$. Then:

Beurling's Theorem ([1]). Let $f$ be bounded and uniformly continuous on $(-\infty, \infty)$. Then there is a real number $\lambda$ and $a$ sequence $\left\{\psi_{n}\right\}$ of linear combinations of translates of $f$, such that

$$
\psi_{n}(x) \rightarrow e^{i \lambda x} \text { narrowly as } n \rightarrow \infty \text {. }
$$

Beurling used complex variable theory in his proof of this theorem. However, it is not hard to see that the main idea behind his reasoning is independent of his use of analytic functions, and a simple demonstration, based on this idea, can be given if one is willing to employ the above theorem on spectral analysis together with a well known

Received September 22, 1964. The preparation of this paper was sponsored by the Office of Naval Research. Reproduction in whole or in part is permitted for any purpose of the United States Government. 
elementary result on synthesis (the same argument thus applies to arbitrary locally compact abelian topological groups). For the reader's convenience, we sketch such a proof now.

Let $f$ be uniformly continuous, and let $K \in L_{1}(-\infty, \infty)$. If, as in [1], one approximates the integral involved in the convolution $K * f$ by finite sums, one sees easily that there is a sequence of linear combinations of translates of $f$ which converges narrowly to $K * f$. (As usual, we write $K * f$ for the function defined by

$$
\left.(K * f)(x)=\int_{-\infty}^{\infty} K(t) f(x-t) d t\right) .
$$

It is therefore enough to show that if $\lambda \in s p f$, there is a sequence of functions $K_{n} \in L_{1}$ such that $\left(K_{n} * f\right)(x) \rightarrow e^{i \lambda x}$ narrowly, $n \rightarrow \infty$. For $h>0$, let $G_{h}$ denote the function in $L_{1}$ whose Fourier transform is zero outside $(\lambda-h, \lambda+h)$, one at $\lambda$, and linear on each of the intervals $[\lambda-h, \lambda],[\lambda, \lambda+h]$. Since $\lambda \in s p f$, we cannot have $G_{h} * f=0$. Let, therefore (and this is Beurling's main idea) $X_{h} \in(-\infty, \infty)$ be such that $\left|\left(G_{h} * f\right)\left(X_{h}\right)\right| \geqq(1-h)\left\|G_{h} * f\right\|_{\infty}$, and put $K_{h}(t)=G_{h}\left(t-X_{h}\right) /\left(G_{h} * f\right)\left(X_{h}\right)$. Then $K_{h} \in L_{1},\left(K_{h} * f\right)(0)=1$, and $\left\|K_{h} * f\right\|_{\infty} \leqq(1-h)^{-1}$ for $h<1$. If we denote by $K$ the function in $L_{1}$ whose Fourier transform is one on $[\lambda-1, \lambda+1]$, zero outside $(\lambda-2, \lambda+2)$ and linear on each of the remaining two intervals, we see that $K * K_{h}=K_{h}$ for $h<1$, so that $K *\left(K_{h} * f\right)=K_{h} * f, h<1$, which, with the previous inequality, implies that the functions $\left(K_{h} * f\right)(x)$ are uniformly equicontinuous for $h<1 / 2$. There is therefore, by Ascoli's theorem, a sequence $h_{n} \rightarrow 0$ such that, if we write $K_{n}=K_{h_{n}}$, the functions $K_{n} * f$ converge uniformly on finite intervals to some continuous function $g$ as $n \rightarrow \infty$. Clearly $\|g\|_{\infty} \leqq 1$, and since $\left(K_{n} * f\right)(0)=1, g(0)=1$, and the convergence of the $K_{n} * f$ to $g$ is in fact narrow.

Now $g(x)=e^{i \lambda x}$. Indeed, $g(0)=1$ and $g$ is continuous, so $s p g$ is not empty by the theorem on spectral analysis. On the other hand,

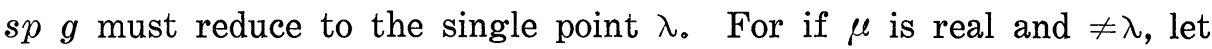
$\varepsilon=1 / 2|\mu-\lambda|$, and let $G$ be the function in $L_{1}$ whose Fourier transform is 1 at $\mu$, zero outside $(\mu-\varepsilon, \mu-\varepsilon)$, and linear on $[\mu-\varepsilon, \mu],[\mu, \mu+\varepsilon]$. Then $G * K_{h}=0$ for all sufficiently small $h$ which, together with the narrow convergence of the $K_{n} * f$ to $g$, implies $G * g=0$, proving $\mu \notin s p g$, since the Fourier transform of $G$ does not vanish at $\mu$. The conclusion $g(x)=e^{i \lambda x}$ now follows by an elementary theorem on spectral synthesis (see, for instance, [4], p. 106 or [7], pp. 151 and 181).

Before going any further we should say that it is clear, from Appendix 1 to [5], that Godement had the above proof in mind when he wrote his paper. He was, however, unable to carry it through because the theorem appealed to at the end of our argument was not then available, except as a corollary of Beurling's reasoning. A general 
demonstration for locally compact abelian topological groups has indeed been given by Domar (see Ch. IV of [3]), but because his exposition is very long and complicated, we have thought it useful to include the above proof.

Now the point we wish to make is that the topology involved in the statement of Beurling's theorem is much stronger than that of the one on spectral analysis. Whereas the second merely asserts that there are functions of the form $e^{i \lambda x}$ in the weak closure of the set of linear combinations of translates of $f$, the first shows that for uniformly continuous $f$, such functions $e^{i \lambda x}$ can be obtained as weak limits of sequences of such linear combinations which are uniformly bounded in norm. Since, for many of the limiting processes used in analysis, the weak closure property is useless without the boundedness condition, it is not entirely correct to say, as some have done (for example, the authors of [4] on p. 106 thereof), that the theorem on spectral analysis contains the gist of Beurling's result. Although the uniform continuity of $f$ is used in an essential way at the beginning of the proof of Beurling's theorem, it is legitimate to ask whether or not one might, by another method, be able to establish some weakened form of it, valid for general $f \in L_{\infty}$, which would still affirm the important boundedness property. One would be satisfied with the following:

Proposition. Let $f \in L_{\infty}$. If $\lambda \in s p f$, the function $e^{i \lambda x}$ belongs to the weak closure of some norm-bounded set of linear combinations of translates of $f$.

Godement recognized the value of such a result, for he states it explicitly in his article (remark on p. 131 of [5]), and uses it to prove Theorem D of that paper. It seems not to have been observed in the literature (neither in Segal's review of Godement's article, written for Mathematical Reviews), that Godement bases the above proposition on an incorrect statement. If $V$ is an arbitrary subset of $L_{\infty}$, closed with respect to the algebraic operation of taking finite linear combinations, let us denote by $[\mathrm{V}]$ the union of the weak closures of all normbounded subsets of $V$. Then Godement establishes the proposition by identifying $[V]$ with the weak closure of $V$, where $V$ is generated by the translates of $f$. However $[V]$ is not in general equal to the weak closure of $V$. (Godement, by the way, cites [2] in support of this claimed equality, but the latter article contains no such affirmation.) It is well to observe here that Godement's proof of Theorem D on $p$. 131 of [5] is in fact wrong on two counts: the use of the above incorrectly established proposition, and, besides this, an additional appeal to the false relation $[[V]]=[V]$, which may fail to hold even when $V$ consists entirely of continuous functions. 
Various subsequent authors (with the exception of Loomis in [7]) have taken one of two positions on the matters we have been discussing: either they assimilate Beurling's result with the theorem on spectral analysis (as in [4], p. 106), or they go to the other extreme and treat the above proposition as an established part of the theory (the author of [8], on p. 8 thereof, seems to claim that the proposition was proved by Beurling in one of his lectures!)

In view of this state of affairs, it is of interest to have a judgement as to the truth of the proposition. It turns out that it is false. The rest of this paper consists of a description of a method for the construction of counterexamples thereto, followed by an indication of our original application of this method. This construction, which is based on an interesting result of Rudin, is merely sketched, because it involves a considerable amount of technical detail, and especially because J.-P. Kahane has found a much simpler one, depending only on the most elementary principles of analysis. Kahane's work is presented in the paper immediately following this one, and the reader may, if he wishes, turn to it as soon as he has read $\S 1$ below.

It should be noted that both Kahane's construction and our original one yield counterexamples to Godement's Theorem D, as well as to the proposition discussed above.

1. If $f \in L_{\infty}(-\infty, \infty)$, we denote by $V_{f}$ the set of finite linear combinations of translates of $f$. The method spoken of at the end of the above discussion in contained in

THEOREM 1. Suppose a function $f \in L_{\infty}(-\infty, \infty)$ has the following properties:

$1^{\circ} \quad 0 \in s p f$.

$2^{\circ}$ If $X_{1}, \cdots, X_{M}$ are distinct real numbers and $A_{1}, \cdots, A_{M}$ are complex, then

$3^{\circ}$

$$
\begin{aligned}
& \sum_{1}^{M}\left|A_{k}\right|=\underset{-\infty<x<\infty}{\operatorname{ess} \sup _{1}}\left|\sum_{1}^{M} A_{k} f\left(x+X_{k}\right)\right| . \\
& \inf _{T>0}\left\{\sup _{-\infty<a<\infty} \frac{1}{T}\left|\int_{a}^{a+T} f(x) d x\right|\right\}=0 .
\end{aligned}
$$

Then 1 is in the weak closure of $V_{f}$, but not in the weak closure of any norm-bounded subset of $V_{f}$.

Proof. Since $0 \in s p f, 1$ is in the weak closure of $V_{f}$.

Let, on the other hand, $E$ be any set consisting of functions $\psi \in V_{f}$ satisfying $\|\psi\|_{\infty} \leqq K<\infty$. Then, if $\psi \in E$, it is of the form 


$$
\psi(x)=\sum_{k} A_{k} f\left(x+X_{k}\right)
$$

with the $X_{k}$ all different, and property 2 implies that $\sum_{k}\left|A_{k}\right| \leqq K$. If $T>0$ is such that

$$
\left|\frac{1}{T} \int_{0}^{T} f(x+X) d x\right|<\frac{1}{2 K}
$$

for all real $X$, we will then have

$$
\left|\frac{1}{T} \int_{0}^{T} \psi(x) d x\right|<\frac{1}{2} \text { for any } \psi \in E .
$$

Since $\frac{1}{T} \int_{0}^{T} 1 \cdot d x=1,1$ cannot be in the weak closure of $E$.

CoRollary 1. If $f \in L_{\infty}$ possesses the properties of the theorem, then there is no sequence of elements of $V_{f}$ which tends weakly to 1 .

For if $\psi_{n} \in V_{f}, n=1,2,3, \cdots$, and the sequence $\left\{\psi_{n}\right\}$ is weakly convergent in $L_{\infty}$, the norms $\left\|\psi_{n}\right\|_{\infty}$ are bounded by a well-known theorem of Banach.

CoROLlary 2. If $f \in L_{\infty}$ is continuous and has the properties of the theorem, it provides a counterexample to Theorem $D$ of [5].

Theorem D of [5] says that if $f \in L_{\infty}$ is continuous and $0 \in s p f$, there is a sequence $\left\{\psi_{n}\right\}$ of elements of $V_{f}$ satisfying $\left\|\psi_{n}\right\|_{\infty} \leqq K$, such that $\psi_{n}(x) \rightarrow 1$ uniformly on finite intervals as $n \rightarrow \infty$. Such a sequence would certainly converge weakly to 1 which is, however, impossible by the theorem.

2. Let us show a way of constructing functions $f$ having the properties required in Theorem 1.

By modifying a construction of Salem, Rudin [10] has shown that there exist perfect sets $P \subset(0,1)$ of Lebesgue measure zero, having the following properties:

I. The elements of $P$ are linearly independent over the rationals.

II. There is a nondecreasing continuous function $F$, defined on $[0,1]$ and constant only on the contiguous intervals of $P$, such that $\int_{P} e^{i \lambda \xi} d F(\xi) \rightarrow 0$ as $\lambda \rightarrow \pm \infty$. 
A detailed exposition of Rudin's construction and the results of Salem on which it is based can be found in Ch. VIII of [6].

By using, if necessary, a set $k P$ instead of $P$, with $k$ suitably chosen $\in(0,1)$ we can ensure, besides properties I and II:

$\mathrm{I}^{\text {bis }}$ The right endpoint of one of the finite intervals contiguous to $P$ is a rational multiple of $\pi$.

It is also easy to see (cf. [6], p. 104) that II in fact implies $\mathrm{II}^{b i s} \int_{J} e^{i \lambda \xi} d F(\xi) \rightarrow 0$ uniformly for all intervals $J$ as $\lambda \longrightarrow \pm \infty$.

Let us denote by $E$ the set obtained from $P$ by removing from it the right endpoint of each of its finite contiguous intervals. $F$ is strictly increasing on $E$ and may, after suitable normalization, be taken to map it in one-to-one fashion onto $[0,1]$. Denote by $\varphi$ the inverse of $F$ restricted to $E ; \varphi$ is strictly increasing on $[0,1]$ and maps that interval onto $E$.

We now define a certain function $f \in L_{\infty}$. First of all, for $n \geqq 2$, we define intervals $I_{n}^{1}, I_{n}^{2}, \cdots, I_{n}^{n}$ as follows:

$$
\begin{aligned}
& I_{n}^{1}=\left[2^{n}, 2^{n}+1\right) \\
& I_{n}^{2}=\left[2^{n}+1,2^{n}+4\right) \\
& I_{n}^{r}=\left[2^{n}+2^{r-1}, 2^{n}+2^{r}\right), \quad 3 \leqq r \leqq n .
\end{aligned}
$$

Denote by $l_{n}^{r}$ the left endpoint of $I_{n}^{r}$, and let $\left\{N_{r}\right\}$ be a rapidly increasing sequence of positive integers. Then, for $x \geqq 4$, put

$$
f(x)=\exp \left[\left(N_{r}+n\right) i \varphi\left(\frac{x-l_{n}^{r}}{\left|I_{n}^{r}\right|}\right)\right] \text { if } x \in I_{n}^{r} .
$$

For $x<4$, let

$$
f(x)=\sum_{1}^{\infty} 2^{-m} \exp \left(\frac{2 \pi i}{2^{m}} x\right)
$$

It is clear that $f \in L_{\infty}$ and $\|f\|_{\infty}=1$.

THEOREM 2. If the sequence $\left\{N_{r}\right\}$ increases rapidly enough, $f(x)$ has properties 1,2 , and 3 of Theorem 1.

Sketch of Proof. In the first place, $f\left(x-2^{M}\right) \rightarrow \sum_{1}^{\infty} 2^{-m} \exp \left(2 \pi i / 2^{m}\right) x$ weakly in $L_{\infty}$ as $N \rightarrow \infty$. Therefore each of the numbers $2 \pi / 2^{m}, m=$ $1,2,3, \cdots$ belongs to $\operatorname{sp} f$ (one way of seeing this is to apply the method use to prove Beurling's theorem in the preliminary discussion of this paper). Since $s p f$ must be closed, we get $0 \in s p f$, establishing 
property 1.

Property 2 follows from the definition of $f(x)$ (for $x \geqq 4$ ) by Kronecker's theorem (see Appendix V of [6]), since $\varphi$ maps [0,1] in one-to-one fashion onto $E$ which forms, together with $\pi$, a set linearly independent over the rationals, according to $I$ and $I^{b i s}$.

To establish property 3 , observe first of all that $I I^{\text {bis }}$ can be restated thus:

$\int_{I} e^{i \lambda \varphi(x)} d x \rightarrow 0$ uniformly for all intervals $I \leqq[0,1]$ as $\lambda \rightarrow \pm \infty$. With this in mind one sees straightforwardly how, looking at the definition of $f(x)$, to assign to $\left\{N_{r}\right\}$ a rate of increase sufficiently rapid so that

$$
2^{-M} \int_{0}^{2^{M}} f(x+a) d x \text { shall tend to zero uniformly in } a \text { as } M \rightarrow \infty \text {. }
$$

COROLLARY. If $\left\{N_{r}\right\}$ increases sufficiently rapidly, Godement's proposition fails for the function $f(x)$.

REMARK. The function $f(x)$ which we have just constructed is not continuous. One can, however, modify the procedure used above so as to obtain a continuous (but not uniformly continuous!) bounded function $g(x)$ which still has properties 1,2 , and 3 of Theorem 1. Essentially, this modification consists in using, on the successive intervals $I_{n}^{r}$, better and better continuous pointwise approximations to the increasing function $\varphi(x)$, instead of $\varphi(x)$ itself. This construction is quite straightforward, but somewhat technical in its details, and we shall not discuss it further. In any event, it leads to a counterexample for Godement's Theorem D.

In conclusion, I would like to thank Professors Arens and Straus for some helpful discussions.

\section{BIBLIOGRAPHY}

1. A. Beurling, Un theoreme sur les fonctions bornees et uniformoment continues sur l'axe reel, Acta Mathematics, 77 (1945), 127-136.

2. J. Dieudonne, La dualite dana les espaces vectoriels topologiques, Annales de l'Ecole Normale Superieure, 59 (1942), 107-139.

3. Y. Domar, Harmonic analysis based on certain commutative Banach algebra, Acta Mathematica, 96 (1956), 1-66.

4. I. Gelfand, D. Raikov, and G. Shilov, Kommutativnye normirovannye kol'tsa, State Publishing House of Physico-Mathematical Literature, Moscow, 1960.

5. S. Godement, Theorems tauberiens et theorie spectrale, Annales de l'Ecole Normale Superieure, 64 (1947), 119-138.

6. J.-P. Kahane, and R. Salem, Ensembles parfaits et series trigonometriques, Fermann, Paris, 1963.

7. L. Loomis, Abstract Harmonic Analysis, Van Nostrand, New York, 1953.

8. B. Nyman, On the One-dimensional Translation Group and Semi-group in Certain 
Function Spaces, Thesis, Uppsala, 1950.

9. W. Rudin, Fourier Analysis on Groups, Interscience, New York, 1962.

10. - Fourier Stieltjes transforms of measures on independent sets, Bull Amer. Math. Soc. 66 (1960), 199-202.

University of CALIFornia, Los ANGELES 


\section{PACIFIC JOURNAL OF MATHEMATICS}

EDITORS

H. SAMELSON

Stanford University

Stanford, California

R. M. BLUMENTHAL

University of Washington

Seattle, Washington 98105
*J. DUGUNDJI

University of Southern California

Los Angeles, California 90007

RICHARD ARENS

University of California

Los Angeles, California 90024

\section{ASSOCIATE EDITORS}

E. F. BECKENBACH

B. H. NeumanN

F. WOLF

K. YosidA

\section{SUPPORTING INSTITUTIONS}

UNIVERSITY OF BRITISH COLUMBIA

CALIFORNIA INSTITUTE OF TECHNOLOGY

UNIVERSITY OF CALIFORNIA

MONTANA STATE UNIVERSITY

UNIVERSITY OF NEVADA

NEW MEXICO STATE UNIVERSITY

OREGON STATE UNIVERSITY

UNIVERSITY OF OREGON

OSAKA UNIVERSITY

UNIVERSITY OF SOUTHERN CALIFORNIA
STANFORD UNIVERSITY

UNIVERSITY OF TOKYO

UNIVERSITY OF UTAH

WASHINGTON STATE UNIVERSITY

UNIVERSITY OF WASHINGTON

AMERICAN MATHEMATICAL SOCIETY CHEVRON RESEARCH CORPORATION

TRW SYSTEMS

NAVAL ORDNANCE TEST STATION 


\section{Pacific Journal of Mathematics}

\section{Vol. 16, No. $1 \quad$ November, 1966}

Larry Armijo, Minimization of functions having Lipschitz continuous first

partial derivatives ............................... 1

Edward Martin Bolger and William Leonard Harkness, Some

characterizations of exponential-type distributions.............. 5

James Russell Brown, Approximation theorems for Markov operators ...... 13

Doyle Otis Cutler, Quasi-isomorphism for infinite Abelian p-groups ...... 25

Charles M. Glennie, Some identities valid in special Jordan algebras but not valid in all Jordan algebras .......................... 47

Thomas William Hungerford, A description of $\operatorname{Mult}_{i}\left(A^{1}, \cdots, A^{n}\right)$ by

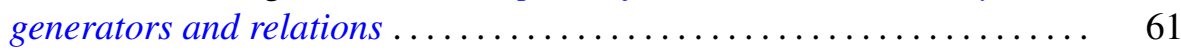

James Henry Jordan, The distribution of cubic and quintic non-residues ....

Junius Colby Kegley, Convexity with respect to Euler-Lagrange differential operators................................... 87

Tilla Weinstein, On the determination of conformal imbedding ......... 113

Paul Jacob Koosis, On the spectral analysis of bounded functions ........ 121

Jean-Pierre Kahane, On the construction of certain bounded continuous functions ................................... 129

V. V. Menon, A theorem on partitions of mass-distribution ........... 133

Ronald C. Mullin, The enumeration of Hamiltonian polygons in triangular

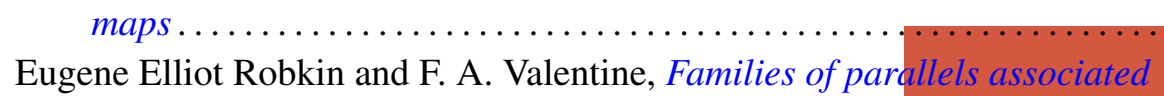

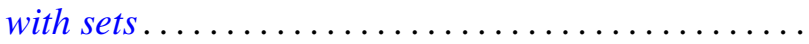

Melvin Rosenfeld, Commutative F-algebras

A. Seidenberg, Derivations and integral closure

S. Verblunsky, On the stability of the set of exponents of a Cauchy

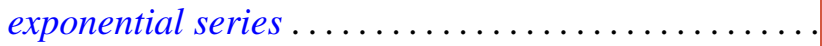

Herbert Walum, Some averages of character sums 